\title{
Our Journal is entering into its second decade
}

\author{
Mart Min \\ 1. Thomas Johann Seebeck Department of Electronics, Tallinn University of Technology, Tallinn, Estonia \\ 2. Section Editor, Journal of Electrical Bioimpedance \\ 3. E-mail any correspondence to: mart.min@taltech.ee
}

\begin{abstract}
The first issue of the Journal of Electrical Bioimpedance saw the light in 2010 by the personal initiative of two men from the University of Oslo, Prof. Sverre Grimnes and Prof. Ørjan G. Martinsen, who has been the editor-in-chief of our Journal during all these ten years. With the sense of gratitude, we hope that he continues his persistent work also during the approaching next decade in the new conditions with a growing number of bioimpedance publications worldwide. However, every success creates new problems, some of which are discussed below.
\end{abstract}

Keywords: Bioimpedance; publications; applications; problems

\section{Present situation}

The tremendous growth of bioimpedance publications the last 30 years has been analyzed by Spanish scientists [1] using the Scopus database (Fig. 1). The same database indexes also the papers of our Journal of Electrical Bioimpedance. In Fig. 2 is drawn a list of institutions with the most extensive rate of publications. True, the figures reflect only the quantity, but also the improvements in content and quality are characteristic to the last decades of biompedance research.

\section{Problems to solve}

The development of instrumentation has led us to wearable and implantable devices enabling continuous monitoring of bioimpedance variations in a wide frequency range. Impedance spectroscopy, tomography and spectro-tomography devices give us a huge amount of data within short time intervals. Although a large amount of data is gathered, less information is available on this and still the lack of knowledge remains obvious. Therefore, implementation of data handling and artificial intelligence methods is urgent.

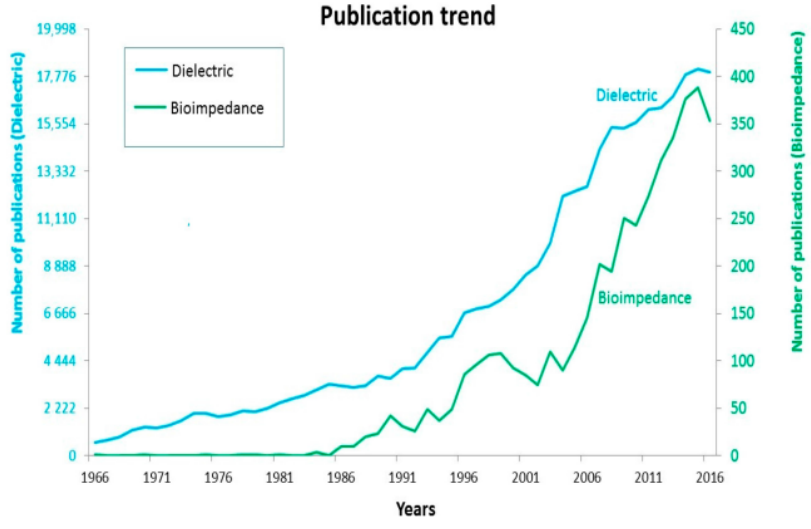

Fig.1: Number of publications on bioimpedance during the last 30 years in comparison with the growth of dielectric impedance papers (extracted from the Scopus based analysis given in [1], published under CC BY 4.0).

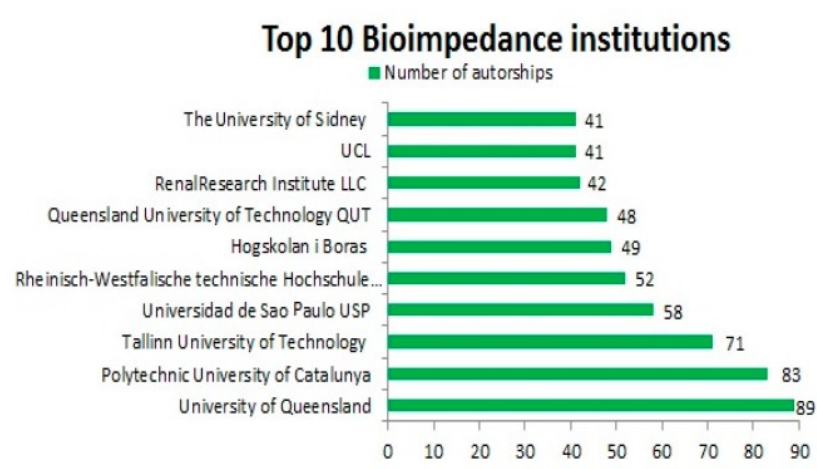

Fig. 2: A graph showing the most active institutions in publishing in the bioimpedance field (extracted from the Scopus based analysis [1], published under CC BY 4.0).

\section{References}

1. El Khaled D, Novas N, Gazquez J-A, Manzano-Agugliaro F. "Dielectric and bioimpedance research studies: A scientometric approach using the Scopus database", MDPI Publication 2018; 6(1): 1-16.

https://doi.org/10.3390/publications6010006 